\title{
Habitat characteristics and metapopulation dynamics of the copepod Tigriopus californicus
}

\author{
Florian Altermatt ${ }^{1,2, *, * *}$, Annette Bieger ${ }^{2,3, * *}$, Steven G. Morgan ${ }^{2}$ \\ ${ }^{1}$ Department of Aquatic Ecology, Eawag: Swiss Federal Institute of Aquatic Science and Technology, Überlandstrasse 133, \\ Dübendorf 8600, Switzerland \\ ${ }^{2}$ Bodega Marine Laboratory, University of California, 2099 Westside Rd., Bodega Bay, California 94923-0247, USA \\ ${ }^{3}$ Institute of Integrative Biology, Swiss Federal Institute of Technology, ETH Zürich, Universitätstrasse 16, Zürich 8092, \\ Switzerland
}

\begin{abstract}
Many organisms live in spatially delimited habitat patches, where local extinctions are compensated by dispersal and recolonization of empty habitat patches. To predict the dynamics of such metapopulations in an era of large environmental changes, it is essential to understand the key abiotic factors affecting local occurrence and temporal variation in patch occupancy. Here, we investigated the metapopulation dynamics of the marine copepod Tigriopus californicus, which is restricted to high intertidal and supralittoral rock pools. We monitored populations of T. californicus in northern California for almost $2 \mathrm{yr}$ and observed pronounced seasonal changes in patch occupancy with $>80 \%$ population turnover annually. Copepod occupancy depended on the area, depth and desiccation of pools and shore height of pools. Most local extinctions were due to pool desiccation, and the pools with an intermediate desiccation risk and low shore heights were the most frequently occupied. We highlight the significance of both the spatial position of pools (shore height) and their ephemerality in explaining occupancy pattern.
\end{abstract}

KEY WORDS: Rock pools $\cdot$ Extinction-colonization dynamics $\cdot$ Dispersal $\cdot$ Occupancy

\section{INTRODUCTION}

Many organisms live in spatially delimited habitat patches that are connected by dispersal. The patchiness of habitats naturally arises from the heterogeneous distribution of key resources across the landscape or derives from anthropogenic habitat fragmentation. Examples of metapopulation dynamics of species inhabiting spatially delimited habitats include aquatic crustaceans and insects dwelling in ponds or tree holes (Kneitel \& Miller 2003, Altermatt \& Ebert 2010), amphibians and fishes residing in ponds or lakes (Sjögren 1991) and marine crustaceans, mussels and fishes inhabiting reefs and tidepools (Pfister 1998, Johnson 2001, Schulte et al. 2009, SaenzAgudelo et al. 2011). Temporal turnover of populations within and among habitat patches is characteristic of all metapopulations (Levins 1970, Hanski \& Gaggiotti 2004), and often, habitat patchiness is intrinsically linked with habitat turnover, resulting in ephemeral habitat patches (Burton 1997, Altermatt et al. 2009). Consequently, each local population is unstable and subject to either random or deterministic extinction. In metapopulations, dispersal and recolonization of patches balance local extinctions and assure longterm persistence across regions (Hanski \& Gaggiotti 2004). To forecast the dynamics of metapopulations in an era of climate change, it is essential to know the key abiotic factors affecting local occurrence and temporal variation in patch occupancy. Potentially, metapopulation dynamics may allow a species to react to climate-change induced changes in habitat availability (O'Connor et al. 2012) and improve long-term persistence. 
Large spatial variation in the size of habitat patches in many metapopulations is an important determinant of occupancy patterns, extinction dynamics and the origin of dispersers (Harrison 1991, Hanski \& Gyllenberg 1993, Crone et al. 2001, Altermatt \& Ebert 2010). Many theoretical models either make assumptions about patch occupancy and patch turnover rates relative to patch size or need to be parameterized with these values from natural systems. Thus, information on these parameters from natural metapopulations is essential. Extinction is generally inversely related to patch size (Harrison 1991), and often, occupancy is higher for larger patches. However, large temporal occupancy fluctuations are known, and the bimodal distributions of site occupancy observed in many species (i.e. the majority of species occur at 1 time either in most to all suitable habitat patches or only in few patches) can be explained using mainland-island metapopulation models that incorporate rescue effects (Hanski \& Gyllenberg 1993). In many empirical systems, it is found, and in most models assumed, that migrants are produced by populations in large patches (Hanski \& Gyllenberg 1993, Gyllenberg \& Hanski 1997), but empirical examples of the predominant origin of migrants from small ephemeral patches are also known (Crone et al. 2001, Altermatt \& Ebert 2010).

Information on occupancy patterns and extinctioncolonization dynamics over time is essential to understand the long-term dynamics and prospects of species. It is known from invertebrate species that metapopulation dynamics can be strongly affected by relatively rare environmental key events, such as annual droughts drying pools (McLaughlin et al. 2002, Altermatt et al. 2008, 2009). Climate warming may increase the desiccation of pools (Altermatt et al. 2009) and accelerate habitat turnover. In a system of planktonic freshwater crustaceans, such climatechange related increases in colonization-extinction dynamics due to desiccation of pools have already been documented (Altermatt et al. 2008, Altermatt \& Ebert 2010), but less is known for marine systems.

Here, we investigated the metapopulation dynamics of the marine copepod Tigriopus californicus, which ranges from Baja California, Mexico, to southeast Alaska, USA. Copepods of the genus Tigriopus are generally restricted to high intertidal and supralittoral rock pools worldwide. These habitats are naturally fragmented with low connectivity among populations, making it an excellent model system to study metapopulation dynamics (Vittor 1971, Dybdahl 1994, Burton 1997, Powlik 1999, Johnson 2001). Desiccation of the habitat causes local extinction, as T. californicus does not have droughtresistant resting stages (Vittor 1971).

In a pioneering study, Dybdahl (1994) examined whether and how metapopulation dynamics affect genetic differentiation among tidepool populations of Tigriopus californicus over 6 to $14 \mathrm{wk}$. By following dozens of populations for $3 \mathrm{mo}$, he found that local extinction rates of populations can be very high: during that time, $\sim 35 \%$ of the populations went extinct, mostly due to desiccation of pools. Furthermore, based on allozyme markers, Dybdahl found that the mean number of colonists arriving in re-inundated pools is $\sim 1.5$ colonists per week. Most of these colonists originated from a subset of local populations, and the genetic diversity in a newly established population increased with the age of the population (Dybdahl 1994). A more recent study by Johnson (2001) looked at population dynamics in a closely related species, T. brevicornis, in pools in the upper and middle-tidal zone over $31 \mathrm{~d}$. This study found that local population dynamics were only weakly correlated in different populations among pools. As already reported by Vittor (1971), population sizes are highly variable throughout the year, but the fluctuations are neither correlated among populations nor correlated with environmental variables, such as salinity or temperature (Vittor 1971). Besides looking at short-term population dynamics, Johnson (2001) also investigated the effect of the shore height of pools on occupancy and population densities. Population declines were especially observed at the lower end of the species range across all shore heights during spring tide (Johnson 2001). Contrary to the generally high extinction rates reported by Dybdahl (1994), Johnson (2001) found relatively few extinction events in pools above the mean high tide level. In summary, these studies showed over a time period of weeks to a few months that (1) local extinction rates can (but not necessarily must) be high (Dybdahl 1994, Johnson 2001) and (2) population dynamics among patches are not correlated (Vittor 1971, Johnson 2001).

The independence of local, within-patch dynamics across all patches is a key assumption of the metapopulation concept (Hanski \& Gaggiotti 2004) and thus an important argument that the herein studied copepods are structured as a metapopulation. While these studies (Dybdahl 1994, Johnson 2001) are, to our knowledge, the first and only looking at metapopulation dynamics in rock pool Tigriopus spp., they did so over a relatively short timescale of 1 to 3 mo, and occupancy and metapopulation dynamics were not related to habitat characteristics. 
While metapopulation dynamics of Tigriopus species have been rarely studied, other aspects of the species' ecology are relatively well understood. For example, populations of $T$. californicus differ in thermal tolerance (Willett 2010, Kelly et al. 2012), showing a latitudinal gradient in adaptation to high temperatures (Willett 2010). Furthermore, the sex ratio within populations is affected by environmental variables, especially temperature (Egloff 1966, Voordouw \& Anholt 2002, Voordouw et al. 2008). Recently, it has also been hypothesized that this species may have a limited capacity to buffer negative effects of climate warming by adapting to higher temperatures due to a relatively low within-population genetic variability in thermal tolerance (compared to the variability between populations) (Kelly et al. 2012). Climate change may not only result in warming of the water, causing heat stress, but may also increase desiccation of pools (Altermatt et al. 2009) and accelerate habitat turnover.

We monitored populations of Tigriopus californicus in northern California periodically for almost $2 \mathrm{yr}$, including extensive droughts and multiple local extinction and colonization events. We determined how patch occupancy is affected by patch size, patch position and environmental stability, especially desiccation, over time. We hypothesized that patch occupancy is an increasing function of patch size and inversely related to the ephemerality of the patch. We also hypothesized that the largest patches would be permanently occupied and serve as source populations.

\section{MATERIALS AND METHODS}

\section{Study area}

We conducted the study on the reserve surrounding the Bodega Marine Laboratory in northern California ( $\left.38^{\circ} 19^{\prime} 03^{\prime \prime} \mathrm{N}, 123^{\circ} 04^{\prime} 23^{\prime \prime} \mathrm{W}\right)$. The coastline is composed of granitic rock, the tides are mixed semidiurnal, and the climate is Mediterranean characterized by summer droughts and winter rains. We mapped 47 pools of various sizes along a $500 \mathrm{~m}$ transect in the high intertidal and supralittoral zones (Fig. 1). The pools were above mean high tide and were replenished by wave splash. Most pools also received freshwater inflow from seasonal seeps of groundwater from adjacent cliffs. In addition to spatial variation in salinity, high evaporation rates result in strongly varying salinity levels in these rock pools over short timescales (Powlik 1999). Furthermore, these high

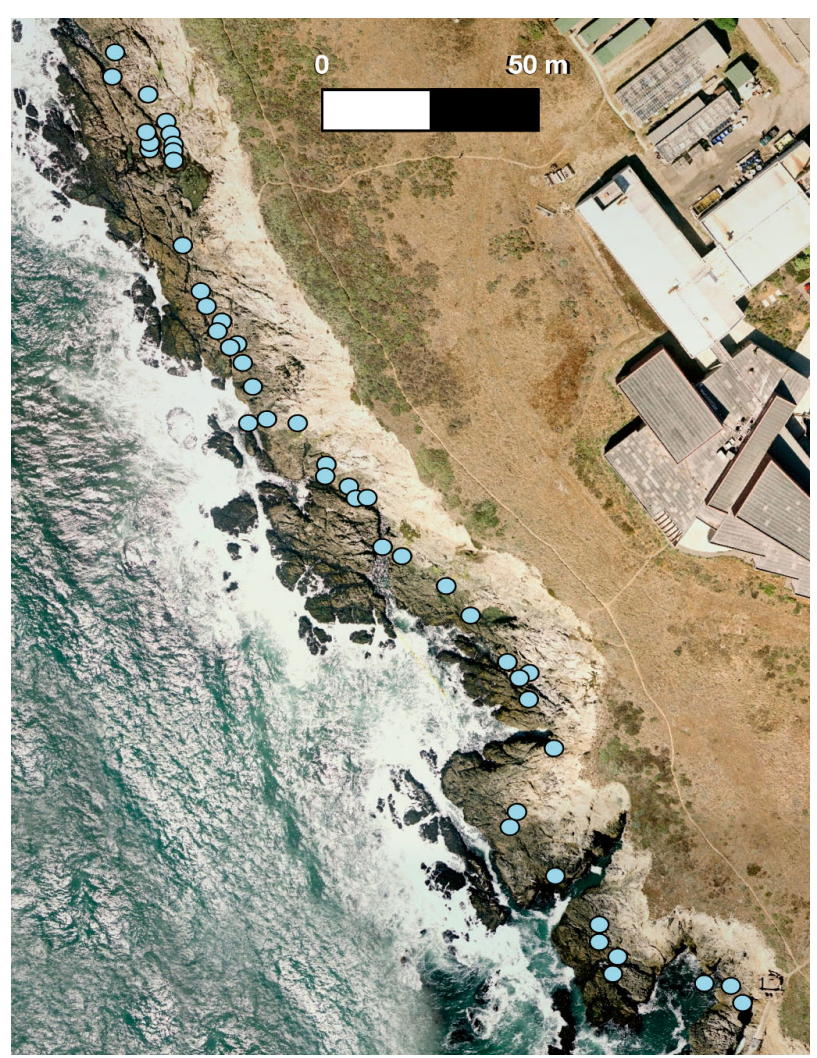

Fig. 1. Location of the 47 rock pools (blue symbols) adjacent to the Bodega Marine Laboratory, northern California, USA. Pools were located in the high intertidal (dark rocks) and supralittoral zones (light rocks) at the Pacific shoreline

intertidal pools are subject to long periods of tidal emersion, experiencing a broad range of diurnal and seasonal variation in temperature (Powlik 1999, Kelly et al. 2012). Due to regular desiccation and strongly fluctuating salinity levels (from freshwater to hypersaline), rock pool communities are depauperate, typically consisting of only ostracods and mosquito larvae (Albert et al. 2001) besides the commonly found harpacticoid copepod Tigriopus californicus.

We measured the maximal surface area and circumference of each pool by taking a digital picture of the pool relative to a meterstick and analyzing the photographs using ImageJ software. At each sampling event, we measured pool depth and recorded desiccated pools. We calculated the proportion of sampling periods that each pool contained water during the study to determine the desiccation likelihood of pools. We also measured the shore height of each pool, using laser level surveying equipment. As a measure of shore height, we chose the height above the mean lowest tide (MLLW). At our study site, the equally relevant mean high water level is on average $1.758 \mathrm{~m}$ above MLLW, the latter being the $0 \mathrm{~m}$ refer- 
ence. As our studied pools are primarily supratidal, the shore height gives the relative height of the pools on the shore as an indicator of desiccation risk.

\section{Sampling}

We surveyed all of the pools 10 times over almost 2 yr $(22 \mathrm{mo})$. Seasonal variation in pool occupancy was determined by sampling 7 times during the first year (13 April, 22 May, 6 August, 3 September, 4 October, 2 November and 2 December in 2010) and 3 times during the second year: before (25 April 2011) and after (26 September 2011) the dry season and once in the middle of the wet season (18 January 2012). Frequent visual inspection of the pools in the second year suggested that there were no further desiccation-inundation cycles that would have allowed additional establishment and subsequent extinction dynamics than the ones observed.

The density of copepods was determined by taking water samples $(250 \mathrm{ml})$ with a large pipette over all depths and microhabitats within each pool. Copepods were transferred into $80 \%$ ethanol upon returning to the laboratory. We counted the number of males, females, ovigerous (egg-bearing) females and juveniles of Tigriopus californicus. We assumed that a population went extinct from a pool if the population had been present during the previous sampling event but we did not find individuals during the subsequent sampling event. In cases in which we did not find copepods in the pipette-based samples, we additionally screened the pool visually for copepods to avoid false-negative observations. Finally, we recorded the presence or absence of freshwater-tolerant ostracods in the pools, which are an indicator of low salinity. The presence or absence of salinity-sensitive organisms provides an integrative measurement of salinity changes and salinity levels over the time between samplings. We corroborated this link by taking occasional temperature and salinity measurements within the pools with a YSI meter.

\section{Analysis}

We used analysis of co-variance (ANCOVA) models to analyze the occupancy rates of copepods (proportion of time present) relative to the envi- ronmental variables surface area, maximal pool depth, desiccation risk, shore height and a quadratic term of shore height. For occupancy, we used the proportion of all of the times a pool was occupied (i.e. irrespective of whether the pool was dry or inundated) as well as the proportion of times occupied while inundated and ran all analyses on both response variables. The former gives 'occupancy sensu lato', and desiccation of the pool will be part of the response variable explained in the model, while the latter gives 'occupancy sensu stricto', only considering the presence of copepods at times when theoretically suitable. The models initially included all main factors and interactions. The residual deviance of the models was used as the goodness-of fit criterion in the model evaluation. The models were hierarchically simplified, using the Akaike information criterion (AIC) as the selection criterion in a stepwise algorithm, starting with removal of highest level interactions first (Crawley 2002). We examined the residual plots from the ANCOVA; the fits were appropriate, and as there was no obvious non-linearity, no transformations were required. We used local polynomial regression fitting (loess-fitting) to visualize the correlation between desiccation risk and occupancy. All analyses were conducted in $R$ Version 2.12.1 (R Development Core Team 2010).

\section{RESULTS}

A pronounced seasonal cycle of copepod abundance and water level occurred (Fig. 2). All pools contained water and many contained copepods in winter, whereas less than $20 \%$ of them did at the end

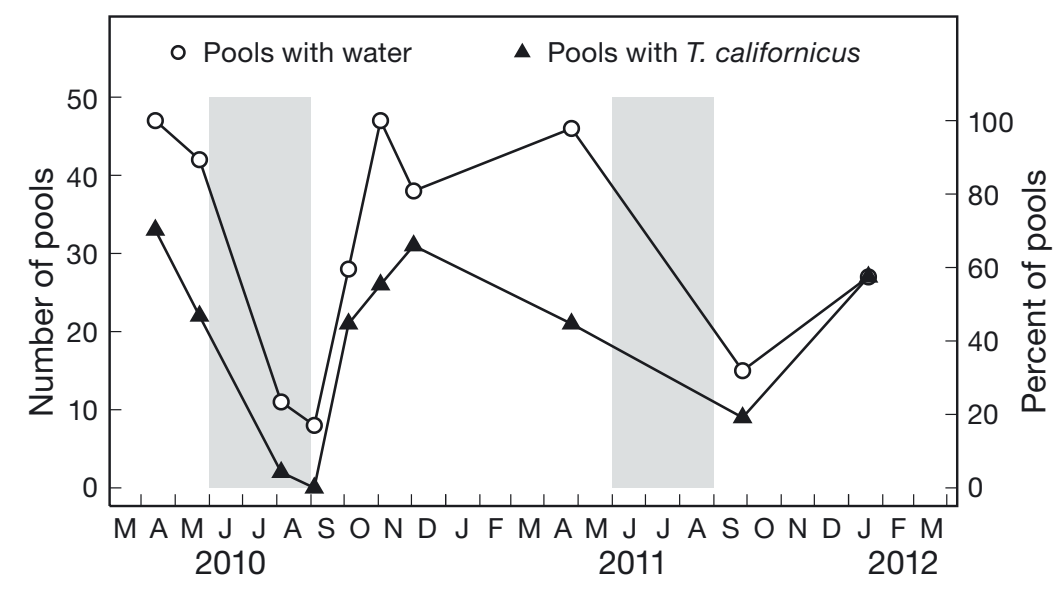

Fig. 2. Temporal dynamics of the number of pools with water and number of pools occupied by Tigriopus californicus over 2 yr. Gray areas indicate the summer drought period, with precipitation $<5 \mathrm{~mm} \mathrm{mo}^{-1}$ 


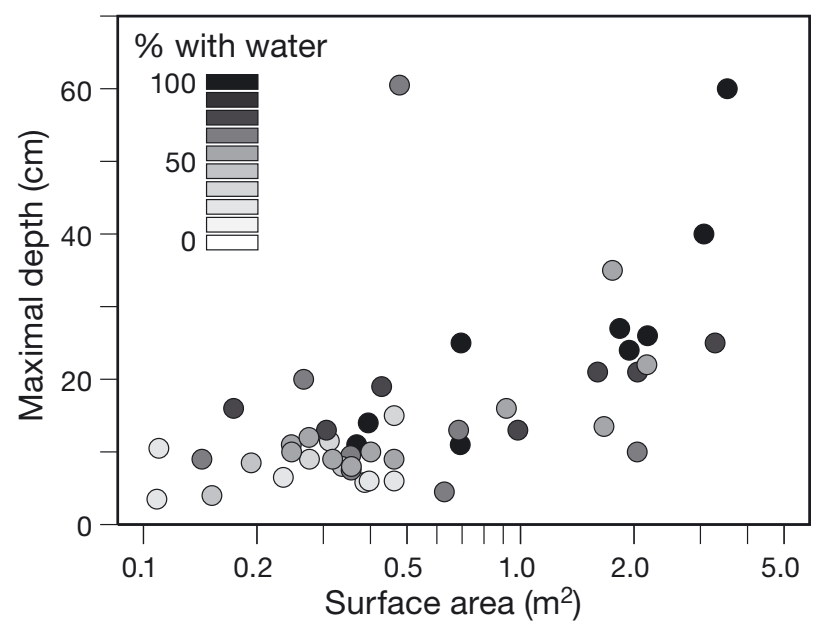

Fig. 3. Percentage of time that a pool contained water (grayscale gradient) relative to pool surface area $\left(\log _{10}\right.$-scale) and maximal pool depth of summer. Small, shallow pools dried up more often than large, deep pools (Fig. 3). The percentage of time dry was explained significantly by the pools' surface area (ANOVA, $F_{1,44}=19.2, \mathrm{p}<0.00001$ ) and the pool's maximal depth (ANOVA, $F_{1,44}=4.2, \mathrm{p}=$ 0.046). The interaction between these 2 naturally covarying pool variables was not significant and was removed from the model.

Overall, copepod occupancy depended significantly on the area, desiccation risk of pools and shore height of pools as well as some interactions among these factors (Fig. 4, Tables 1 \& 2). The models explained $56 \%$ and $51.4 \%$ of the variance in occupancy patterns (occupancy sensu lato and sensu stricto, respectively; Tables 1 \& 2). Depending on whether the model was explaining occupancy sensu lato or sensu stricto, the order of variance explained by the different factors was slightly different. For occupancy sensu lato, des-
Fig. 4. Proportion of time a pool was occupied by Tigriopus californicus relative to the pool's desiccation risk and the pool's shore height. $(\mathrm{A}, \mathrm{B})$ Occupancy sensu lato, i.e. pools occupied at all times; (C,D) occupancy sensu stricto, where pools were only considered when inundated. Dots are scaled relative to the pools' surface area. Black lines are loess-smoothed fits of occupancy relative to desiccation risk and shore height, respectively
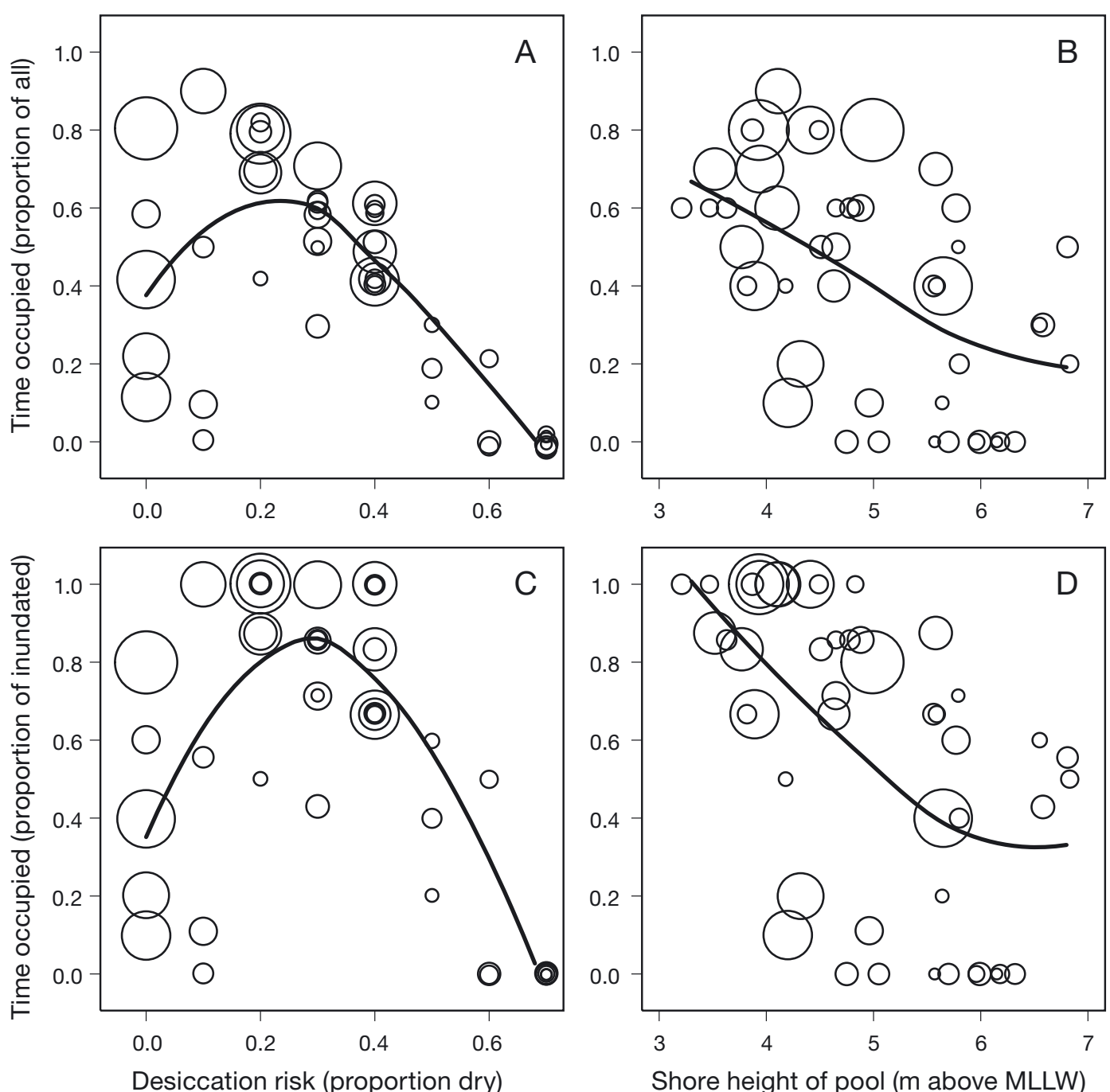

Shore height of pool ( $\mathrm{m}$ above MLLW) 
Table 1. ANCOVA model explaining occupancy sensu lato (proportion of all time that a pool was occupied by Tigriopus californicus) by pool surface area, desiccation risk, maximal pool depth, shore height and interactions among these factors retained in the model. SSQ: sum of squares; Mean SQ: mean squares

\begin{tabular}{|lcccrcc|}
\hline Estimate & df & SSQ & Mean SQ & F-value & p-value \\
\hline Pool surface area & 1 & 0.54 & 0.54 & 13.7 & 0.0006 \\
Desiccation risk & 1 & 0.63 & 0.63 & 16.1 & 0.0003 \\
Max. pool depth & 1 & 0.01 & 0.01 & 0.1 & 0.7 \\
Shore height & 1 & 0.54 & 0.54 & 13.7 & 0.0006 \\
Pool surface area $\times$ Desiccation risk & 1 & 0.12 & 0.12 & 3.1 & 0.084 \\
Pool surface area $\times$ Max. pool depth & 1 & 0.16 & 0.16 & 4.0 & 0.051 \\
Residuals & 40 & 1.58 & 0.04 & & \\
\hline
\end{tabular}

Table 2. ANCOVA model, explaining occupancy senus stricto (proportion of time that an inundated pool was occupied by Tigriopus californicus) by pool surface area, desiccation risk, maximal pool depth, shore height and interactions among these factors retained in the model. SSQ: sum of squares; Mean SQ: mean squares

\begin{tabular}{|lcccccc|}
\hline Estimate & df & SSQ & Mean SQ & F-value & p-value \\
\hline Pool surface area & 1 & 0.42 & 0.42 & 5.4 & 0.025 \\
Desiccation risk & 1 & 0.62 & 0.62 & 8.0 & 0.007 \\
Max. pool depth & 1 & 0.04 & 0.04 & 0.5 & 0.5 \\
Shore height & 1 & 1.41 & 1.41 & 18.2 & 0.0001 \\
Pool surface area $\times$ Desiccation risk & 1 & 0.46 & 0.46 & 6.0 & 0.019 \\
Pool surface area $\times$ Max. pool depth & 1 & 0.32 & 0.32 & 4.2 & 0.048 \\
Residuals & 40 & 3.10 & 0.08 & & \\
\hline
\end{tabular}

pancy sensu stricto, shore height explained most of the variance in occupancy $(22.1 \%)$, while desiccation risk ( $9.7 \%$ variance explained) and pool surface area $(6.6 \%$ variance explained) contributed less. Again, pool depth was negligible ( $0.6 \%$ variance explained). In both models, all interaction terms retained in the models together accounted for relatively little of the variance $(7.9$ and $12.3 \%$, respectively). Pools with an intermediate desiccation risk were the most frequently occupied (Fig. 4A,C), whereas pools with either a very low or very high desiccation risk were the least occupied. On average, pools at lower shore height were more often occupied than pools at higher shore height (Fig. 4B,D). Occupancy was higher in larger pools, except for a few large pools at relatively low shore height. Interestingly, there was a significant effect of the interaction between pool surface area and desiccation risk on occupancy iccation risk (17.7\% variance explained), pool surface area $(15.1 \%$ variance explained) and shore height (15.1\% variance explained) contributed about equally to the variance explained, while pool depth was negligible $(0.2 \%$ variance explained). For occu-

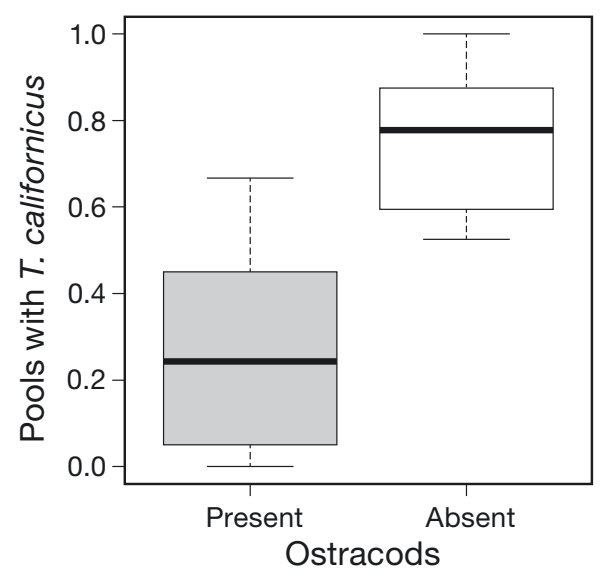

Fig. 5. Proportion of pools occupied by Tigriopus californicus where ostracods were present (gray box) and absent (white box) over the 2 yr study. Lower and upper box-limits are the $25 \%$ and $75 \%$ percentile, respectively. Midline is the median. Whiskers extend to 1.5 times the interquartile range from the box
(Table 2), meaning that while occupancy generally increased with increasing pool size and decreased desiccation risk, there were exceptionally low occupancy rates in a few large pools with low desiccation risk (large pools with low desiccation risk and low occupancy in bottom right corners of the panels in Fig. 4A,C).

Finally, we observed across the 10 sampling events that the occurrence of the freshwater-sensitive copepods and the freshwater-tolerant ostracods was not random. Tigriopus californicus was found significantly less often in pools with ostracods than pools without ostracods (paired Wilcoxon signed-rank test, $\mathrm{V}=0, \mathrm{p}=0.016$; Fig. 5).

\section{DISCUSSION}

We observed strong seasonal dynamics in the occupancy patterns of the marine copepod Tigriopus californicus, with non-trivial effects of habitat characteristics on the pool-specific occurrence of the species. The pronounced seasonal changes in patch occupancy (Fig. 2) suggest an annual turnover of $>80 \%$ of the populations. Pool size, desiccation risk and pool 
shore height affected occupancy (Fig. 4, Tables 1 \& 2). Because T. californicus does not have a drought resistant resting stage (Vittor 1971), as do ostracods and some other crustaceans, the commonly observed desiccation of individual rock pools (Figs. $2 \& 3$ ) is the most likely reason for local extinction (Vittor 1971, Dybdahl 1994). However, some large, permanent pools also had low occupancy (resulting in significant interaction terms; Tables 1 \& 2). Our findings thus show that metapopulation models commonly making simple and linear relationships between environmental variables and occupancy patterns or suitability of patches (Hanski \& Gyllenberg 1993, Hanski \& Gaggiotti 2004) may not be realistic for some natural metapopulations. While increasing patch size and increasing patch stability can be good predictors of occupancy (Harrison 1991, Hanski \& Gyllenberg 1993, Hanski \& Gaggiotti 2004) and are commonly used this way in metapopulation models, we found that their effects are not independent but interact in the herein studied T. californicus metapopulation. Our results confirm previous studies on different taxa (freshwater crustaceans and voles), which showed that patches with medium to high instability can be an important habitat type and a potentially important source of migrants (Crone et al. 2001, Altermatt et al. 2011).

As expected, the smaller pools dried up most often (Fig. 3). Occupancy generally decreased with increasing desiccation risk (Fig. 4A,C) and increasing shore height of pools (Fig. 4B,D). The shore height of pools restricts the inflow of seawater and thereby increases the likelihood of desiccation. The majority of pools that fell below the loess-fit line in Fig. 4B were pools that were not only small in surface area but also rather shallow. The small volume of these pools made them prone to desiccation and possibly also resulted in strong temperature changes. Such temperature changes in small pools are known from other rock pool systems as well (Altermatt \& Ebert 2008, Altermatt et al. 2009) and may be detrimental for Tigriopus californicus populations as the species has a limited thermal tolerance (Willett 2010). The very high population turnover rates highlight the importance of dispersal for continuous recolonization of empty habitat patches. Evaporation and pool desiccation is even expected to increase in the context of climate change (Altermatt et al. 2009).

Surprisingly, some medium to large size pools with very low desiccation risk also had low to very low occupancy (Fig. 4A,C), and pools of intermediate ephemerality had the highest occupancy. A possible explanation for the low occupancy in more perma- nent (large) and saline pools is the high predation risk in these localities (Dethier 1980). Generally, these pools are positioned at lower shore height or are big enough to never dry up (Figs. $2 \& 3$ ). These more permanent pools generally harbor more predators, such as sculpins, anemones or mussels (Vittor 1971, Dethier 1980). Another category of permanent pools had freshwater seeping into the pools, thereby preventing desiccation but at the same time also reducing salinity and making the pools less suitable for Tigriopus californicus. Indeed, fewer pools contained T. californicus when freshwater-tolerant ostracods were present than when they were absent (Fig. 5). Spot-checked salinity measures confirmed that the range of salinities in these pools was low $(0.2$ to $3.5 \mathrm{psu}$ ).

With a generation time of 2 to 4 wk (Dybdahl 1994, Kelly et al. 2012), extinction and recolonization dynamics occur over time-spans including 2 to 6 generations. While we did not directly measure dispersal, genetic data from other studies suggest that recolonization of pools mostly comes from copepods dispersing from a few permanent populations in the upper intertidal zone during high tide and large wave events (Dybdahl 1994). Even though waves are often large and currents are strong in this persistent upwelling region, pronounced genetic differentiation among populations occurs at a localized scale (Burton \& Lee 1994, Dybdahl 1994, Burton 1997). Based on genetic data, these studies also found that dispersal happens mostly across populations within individual rocky outcrops and that there is little dispersal across stretches of sandy beaches, even at the scale of a few dozen to hundred meters of shore lengths. Across stretches of sandy beaches, no tidepools exist that could function as stepping-stones, and so dispersal is low. However, in our study area (Fig. 1), we did not have such large intermittent stretches of sandy beaches. Low dispersal may also result from these benthic copepods holding on to the substrate during storms or predation of this conspicuously colored species by sculpins and other predators lower in the intertidal zone (Dethier 1980).

Our findings highlight the necessity of including multiple environmental variables to understand occupancy patterns and metapopulation dynamics. Individual factors such as patch size (in our case, pool area), patch stability (in our case, pool surface area) and patch quality (in our case, shore height, as a measure of salinity and presence of marine predators) all explained roughly similar proportions of variance of 15 to $18 \%$ of the total variance in overall occupancy (occupancy sensu lato; Table 1). Also, 
when looking at occupancy sensu stricto, only considering patches at times when inundated and thus theoretically available for aquatic organisms, the proportion of variance explained by these variables was relatively similar, with shore height being most important. Obviously, and similar to many other natural systems, the observed environmental variables are not independent of each other (Fig. 3). Larger pools have a lower desiccation risk, but then this also depends on the shore height position. Pools closer to the sea have a lower desiccation risk due to the inflow of sea water, but more predators may be present as well. Such correlations of environmental variables are common, and many metapopulation or metacommunity models have been used to address the effect of spatial or temporal autocorrelations of environmental factors (Vuilleumier et al. 2007, Elkin \& Possingham 2008, Büchi et al. 2009). Recent experimental work is showing that the intrinsic links of environmental factors (e.g. spatial correlation of patch quality and patch stability) are causing nontrivial occupancy patterns and affecting overall diversity patterns (Altermatt \& Holyoak 2012). The Tigriopus system may be a good natural example of such a scenario, in which the outcome (i.e. occupancy of a patch; Fig. 4) is the result of a complex, multifactorial and interacting process, influenced by patch size, patch quality and patch stability.

In conclusion, we monitored patch occupancy dynamics of Tigriopus californicus over a period of almost $2 \mathrm{yr}$, covering multiple local extinction and colonization events. We found pronounced cyclical occupancy patterns that were most likely driven by pool desiccation. Occupancy and sex ratio were correlated with the surface area, depth and shore height of pools. In contrast to previous work on the occupancy of Tigriopus in tidepools, we highlight the significance of both the shore height and ephemerality of pools due to desiccation. The ephemerality of the habitat patches and the high population turnover make T. californicus an excellent model species for studying natural metapopulations.

Acknowledgements. F.A. and A.B. thank the staff of the Bodega Marine station for all their hospitality. J. Sones and S. Gravem helped with fieldwork, especially with the GPS and tidal height measurements. We thank M. Kelly for discussions on Tigriopus. This publication is a contribution of the Bodega Marine Laboratory, University of California at Davis. F.A. was supported by the Swiss National Science Foundation (grant no. PBBSP3-124435), and S.G.M. was supported by the US National Science Foundation (OCE092735).

\section{LITERATURE CITED}

Albert AYK, Borkent CJ, Duquette SL, Voordouw MJ, Anholt BR (2001) Effects of an introduced mosquito on juvenile Tigriopus californicus (Copepoda: Harpacticoidea) in supratidal pools. Arch Hydrobiol 152:203-213

Altermatt F, Ebert D (2008) The influence of pool volume and summer desiccation on the production of the resting and dispersal stage in a Daphnia metapopulation. Oecologia 157:441-452

Altermatt F, Ebert D (2010) Populations in small, ephemeral habitat patches may drive dynamics in a Daphnia magna metapopulation. Ecology 91:2975-2982

Altermatt F, Holyoak M (2012) Spatial clustering of habitat structure effects patterns of community composition and diversity. Ecology 93:1125-1133

Altermatt F, Pajunen VI, Ebert D (2008) Climate change affects colonisation dynamics in a metacommunity of three Daphnia species. Glob Change Biol 14:1209-1220

- Altermatt F, Pajunen I, Ebert D (2009) Desiccation of rock pool habitats and its influence on population persistence in a Daphnia metacommunity. PLoS ONE 4:e4703

Altermatt F, Bieger A, Carrara F, Rinaldo A, Holyoak M (2011) Effects of connectivity and recurrent local disturbances on community structure and population density in experimental metacommunities. PLoS ONE 6:e19525

Büchi L, Christin PA, Hirzel AH (2009) The influence of environmental spatial structure on the life-history traits and diversity of species in a metacommunity. Ecol Model 220:2857-2864

Burton RS (1997) Genetic evidence for long term persistence of marine invertebrate populations in an ephemeral environment. Evolution 51:993-998

Burton RS, Lee BN (1994) Nuclear and mitochondrial gene genealogies and allozyme polymorphism across a major phylogeographic break in the copepod Tigriopus californicus. Proc Natl Acad Sci USA 91:5197-5201

Crawley MJ (2002) Statistical computing: an introduction to data analysis using S-plus. John Wiley \& Sons, Chichester

Crone EE, Doak D, Pokki J (2001) Ecological influences on the dynamics of a field vole metapopulation. Ecology 82: 831-843

Dethier MN (1980) Tidepools as refuges: predation and the limits of the harpacticoid copepod Tigriopus californicus (Baker). J Exp Mar Biol Ecol 42:99-111

$>$ Dybdahl MF (1994) Extinction, recolonization, and the genetic structure of tidepool copepod populations. Evol Ecol 8:113-124

Egloff DA (1966) Ecological aspects of sex ratio and reproduction in experimental and field populations of the marine copepod Tigriopus californicus. Stanford University Press, Palo Alto, CA

- Elkin CM, Possingham H (2008) The role of landscapedependent disturbance and dispersal in metapopulation persistence. Am Nat 172:563-575

Gyllenberg M, Hanski I (1997) Habitat deterioration, habitat destruction, and metapopulation persistence in a heterogenous landscape. Theor Popul Biol 52:198-215

Hanski I, Gaggiotti OE (eds) (2004) Ecology, genetics and evolution of metapopulations. Elsevier Academic Press, Amsterdam

> Hanski I, Gyllenberg M (1993) Two general metapopulation models and the core-satellite species hypothesis. Am Nat 142:17-41 
Harrison S (1991) Local extinction in a metapopulation context: an empirical evaluation. Biol J Linn Soc 42:73-88

Johnson MP (2001) Metapopulation dynamics of Tigriopus brevicornis (harpacticoida) in intertidal rock pools. Mar Ecol Prog Ser 211:215-224

Kelly MW, Sanford E, Grosberg RK (2012) Limited potential for adaptation to climate change in a broadly distributed marine crustacean. Proc R Soc Lond B Biol Sci 279:349-356

Kneitel JM, Miller TE (2003) Dispersal rates affect species composition in metacommunities of Sarracenia purpurea inquilines. Am Nat 162:165-171

Levins R (1970) Extinction. In: Gerstenhaber M (ed) Some mathematical problems in biology. American Mathematical Society, Providence, RI, p 77-107

McLaughlin JF, Hellmann JJ, Boggs CL, Ehrlich PR (2002) Climate change hastens population extinctions. Proc Natl Acad Sci USA 99:6070-6074

O'Connor MI, Selig ER, Pinsky M, Altermatt F (2012) Toward a conceptual synthesis for climate change responses. Glob Ecol Biogeogr 21:693-703

Pfister CA (1998) Extinction, colonization, and species occupancy in tidepool fishes. Oecologia 114:118-126

Powlik JJ (1999) Habitat characters of Tigriopus californicus (Copepoda: Harpacticoida), with notes on the dispersal of supralittoral fauna. J Mar Biol Assoc UK 79:85-92

R Development Core Team (2010) R: a language and environment for statistical computing, Version 2.12.1. R Foundation for Statistical Computing, Vienna

Editorial responsibility: Anna Pasternak, Moscow, Russian Federation
Saenz-Agudelo P, Jones GP, Thorrold SR, Planes S (2011) Connectivity dominates larval replenishment in a coastal reef fish metapopulation. Proc R Soc Lond B Biol Sci 278:2954-2961

Schulte DM, Burke RP, Lipcius RN (2009) Unprecedented restoration of a native oyster metapopulation. Science 325:1124-1128

Sjögren P (1991) Extinction and isolation gradients in metapopulations: the case of the pool frog (Rana lessonae). Biol J Linn Soc 42:135-147

Vittor BA (1971) Effects of the environment on fitnessrelated life history characters in Tigriopus californicus. $\mathrm{PhD}$ thesis, University of Oregon, Eugene, OR

> Voordouw MJ, Anholt BR (2002) Environmental sex determination in a splash pool copepod. Biol J Linn Soc 76: 511-520

Voordouw MJ, Stebbins G, Robinson HE, Perrot-Minnot MJ, Rigaud T, Anholt BR (2008) Genetic variation in the primary sex ratio in populations of the intertidal copepod, Tigriopus californicus, is widespread on Vancouver Island. Evol Ecol Res 10:1007-1023

> Vuilleumier S, Wilcox C, Cairns BJ, Possingham HP (2007) How patch configuration affects the impact of disturbances on metapopulation persistence. Theor Popul Biol 72:77-85

Willett CS (2010) Potential fitness trade-offs for thermal tolerance in the intertidal copepod Tigriopus californicus. Evolution 64:2521-2534

Submitted: March 13, 2012; Accepted: August 10, 2012 Proofs received from author(s): October 30, 2012 